Article

\title{
Meeting Boundaries: Exploring the Faces of Social Inclusion beyond Mental Health Systems
}

\author{
Carole Heather Walker * and Sophie Thunus \\ Health and Society Institute, UCLouvain, 1200 Brussels, Belgium; E-Mails: carole.walker@uclouvain.be (C.H.W.), \\ sophie.thunus@uclouvain.be (S.T.) \\ * Corresponding author
}

Submitted: 13 April 2019 | Accepted: 28 November 2019 | Published: 20 March 2020

\begin{abstract}
This article examines social inclusion in the context of the deinstitutionalisation of mental health care. It draws on a scientific evaluation of the Belgian reform of mental health care (2010), designed to assess the influence of organisational mechanisms on the social and care trajectories of service users. The findings highlight the ongoing challenge for mental health systems to support the inclusion of service users within the community, and the increasingly difficult access to mental health care for people with complex and chronic mental health problems. Drawing from Systems Theory (Luhmann, 2013) and the analysis of subjective experiences, this article delves into the complex processes of social inclusion using the empirically-grounded concepts of the patient role and the impatient role. By acknowledging the relational dimensions of social inclusion, this article argues that complementarities between two faces of the mental health system are key to achieving inclusion beyond the walls of institutions and within society at large.
\end{abstract}

\section{Keywords}

deinstitutionalisation; ethnography; mental health care; social inclusion; systems theory

\section{Issue}

This article is part of the issue "Institutions of Inclusion and Exclusion" edited by J. Cok Vrooman (Utrecht University and The Netherlands Institute for Social Research|SCP, the Netherlands) and Marcel Coenders (Utrecht University and The Netherlands Institute for Social Research|SCP, the Netherlands).

(C) 2020 by the authors; licensee Cogitatio (Lisbon, Portugal). This article is licensed under a Creative Commons Attribution 4.0 International License (CC BY).

\section{Introduction}

Mental health care systems across European countries have undergone major changes since the mid-twentieth century. Growing public criticism of psychiatric hospitals drove policy makers to place increasing emphasis on the inclusion of people with mental health problems within society. The deinstitutionalisation of mental health care gradually gained international consensus, and efforts began to shift mental health care from hospitals to the community.

Despite the development of community mental health care, scientific evaluations carried out internationally have revealed undesirable side effects. People with complex and chronic mental health problems (PCCs) now face difficult access to mental health care (Novella, 2010). In the Belgian context, these evaluations have repeatedly pointed to excessive recourse to long-term hospital stays for those admitted. On a broader level, there is still an urgent need to facilitate social inclusion of people with mental health problems outside mental health institutions and within the community (Grard, Nicaise, \& Lorant, 2015).

This article aims to address the complex relationship between the deinstitutionalisation of mental health care and social inclusion. It draws on a research project designed to assess the Belgian reform of mental health care (2010) and its impact on the social and care trajectories of mental health service users (Walker, Nicaise, \& Thunus, 2019). The research results shed new light on the pro- 
cess of social inclusion for mental health service users. The qualitative data substantially widens the scope of social inclusion, often limited to citizens' access to services available within a given community, as it incorporates personal experiences of social involvement as fullfledged members of society. Drawing from these findings, this article raises two questions. First, how do contemporary mental health systems contribute to the process of social inclusion for people with mental health problems? Secondly, how can PCCs navigate towards social inclusion, in the context of their increasing exclusion from the mental health system's services?

This article refers to Systems Theory (Luhmann, 2013) in order to discuss the complex relationship between the mental health system and social inclusion. This theoretical framework is particularly relevant in tackling these complexities, as it draws a distinction between different types of systems which include social systems and psychic systems, each based on specific "modes of operation" (Luhmann, 2013, p. 188). In addition, it theorises the process through which these systems interact with one another and relate to their environment. The empirical contribution incorporates service users' subjective experiences of social inclusion. It highlights the performance of two roles, the patient role and the impatient role, and the contribution of the contexts created by different social systems in shaping these performances. Both roles are ideal types, each capturing distinctive characteristics of speeches and interactions, and leading to different pathways towards social inclusion.

By disentangling the concept of social inclusion in relation to mental health systems, this article brings the relational dimensions of social inclusion into the discussion, thereby addressing the complementarities between two faces of the mental health system: the mental health system per se and a system made up of alternative services. This article argues that these complementarities are key to achieving inclusion for people with mental health problems beyond the boundaries of mental health systems and within society at large.

\section{The Two Faces of the Belgian Mental Health System}

During the mid-twentieth century, psychiatric hospitals were strongly criticised for dehumanising patients and cutting them off from society. Social pressure encouraged a process of deinstitutionalisation and the World Health Organization supported the promotion of community mental health care (Sturdy, Freeman, \& Smith-Merry, 2013), which values the emancipation of people with mental health problems. European countries began to launch reforms of mental health care which took various forms according to national specificities and institutional arrangements (Thunus \& Schoenaers, 2017). They all had in common the emphasis placed on prevention, rehabilitation, and social inclusion. Overall, the deinstitutionalisation process unfolded in light of the synergy between social movements on the one hand, and the formal mental health system on the other.

In Belgium, the anti-psychiatric movement played a key role in promoting patient autonomy and social inclusion as important ideals among policy makers and mental health professionals. As of the early 1960s, these ideals inspired the creation of non-profit associations that developed innovative practices (Thunus, 2015). In turn, these inspired reforms in mental health care that were being launched at the same time (Bergeron \& Castel, 2016; Feys, 2017).

After the first two reforms of mental health care, certain non-profit associations were included in the mental health system. Others remained external, but gradually started to collaborate with mental health services. In this article, these external services will be referred to as alternative services. Importantly, these do not form a homogeneous group, particularly as they developed across different sectors (e.g., health promotion and continuing education), they are subsidised differently, and they are subject to different regulations. By and large, alternative services offer alternative responses to mental health care without, however, objecting to the mental health system's formal services. The alternative nature of their approach is described in the following sections.

The Belgian mental health system, per se, first developed in 1948, when mental health was transferred from the judicial to the healthcare jurisdiction. This resulted in a rapid professionalisation and medicalisation of psychiatric hospitals (de Munck, Genard, Olgierd, Vrancken, \& Delgoffe, 2003). The first mental health reform was launched in 1975 with the creation of community mental health services, defined as facilities for people with a wider variety of disorders. These services substantially diversified the mental health system's activities and enlarged its target population (Verhaegen, 1987).

The second reform of mental health care (1989) introduced Sheltered Housing (SH) and Psychiatric Nursing Homes (PNH). SH offers transitory accommodation where "activities are organised and support is provided to help residents acquire appropriate social skills" (Gerkens et al., 2010, p. 194). It abides by psychiatric rehabilitation principles and is geared towards social autonomy. PNH intend to provide "supportive permanent care for chronic psychiatric patients whose symptoms are stabilized" (Hermans, de Witte, \& Dom, 2012, p. 289). In theory, both types of facilities conformed to the emancipatory ideals promoted by non-profit associations. However, it quickly emerged that $\mathrm{SH}$ and $\mathrm{PNH}$ were reproducing what psychiatric hospitals had initially been criticised for. Indeed, patients who no longer required intensive treatments were still not reintegrating in their home and local communities (de Munck et al., 2003).

Together, the first two reforms of mental health care supported the process of deinstitutionalisation. However, social inclusion beyond the walls of mental health institutions was still insufficient and recourse to 
psychiatric hospitals remained frequent (Grard et al., 2015). Indeed, the total number of psychiatric beds in Belgium remained too high in comparison to other European countries (World Health Organization, 2018).

In order to meet these challenges, the third reform of the Belgian mental health system was launched in 2010. The '107-reform,' named after a Hospital Act (art. 107) its funding is based on, was initiated through exploratory projects addressed to the general population. Each project was designed to create local networks in which services collaborate to provide five care functions: prevention and promotion; mobile teams; social rehabilitation; intensive psychiatric care; and alternative housing facilities (Federal Service for Public Health, 2010).

The 107-reform was intended to be comprehensive, and to include within each network not only all services belonging to the mental health system (the 'first circle'), but also alternative and social services (the 'second circle') that place the emphasis on service users' personal autonomy and social inclusion.

Despite efforts to overcome the challenges encountered during the previous reforms, scientific evaluations of the 107-reform highlighted major shortcomings. Indeed, alternative and social services (the 'second circle') were poorly integrated within the local mental health networks. This hindered the socio-professional reinsertion of mental health service users, and even exacerbated PCCs problems of accessibility to mental health care (Grard et al., 2015). These shortcomings have been associated with the increasing formalisation of admission procedures, a result of the collaborative networks' development (Walker et al., 2019). Furthermore, psychiatric hospitals' involvement in the reform's financing mechanisms has also played a role (Thunus, 2015).

\section{Methods}

This article draws on a research evaluation of the Belgian mental health care reform launched in 2010. This qualitative research was commissioned by Regional authorities in Brussels and was carried out in 2018 over a period of nine months. Its aim was to assess the influence of organizational mechanisms on service users' social and care trajectories, and to formulate policy recommendations drawing from this analysis. The research was designed to incorporate the main challenges since the reform's implementation in 2010, which include difficult access to mental health care for PCCs.

The research design consisted of four phases: (1) semi-structured interviews with network coordinators and service managers; (2) focus groups with current or former mental health service users; (3) focus groups with professionals; and (4) ethnographic fieldwork carried out in alternative services. Participants were recruited using a snowball sampling procedure through the boards of hospitals and ambulatory services, coordinators of mental health care networks, and service user organizations. The final selection of participants was made to include a wide diversity of sectors, organizations, professional disciplines, and functions. The research protocol was submitted to an Ethical Committee at UCL Saint-Luc that marked its approval, and all participants gave their consent to take part in the research.

The four phases were each associated with an intermediary objective. First, the semi-structured interviews aimed to gain insight into work and collaborative practices, as well as attitudes towards the reform's political objectives. Second, the focus groups with service users aimed to identify significant turning points within their respective social and care trajectories. The discussions raised key themes which were further explored in phase three involving separate focus groups with professionals who have direct contacts with mental health service users in their practice.

The ethnographic fieldwork was added to the initial research design in order to allow for a more diversified sample of PCCs. Indeed, few people with a history of difficult access to mental health care-either due to exclusion from services available or to their own rejection of these services-volunteered to take part in the focus groups. In fact, most volunteers were representatives involved in service user organizations. Thus, both authors carried out ethnographic fieldwork in alternative services, which are not formally part of the mental health system. The explicit aim of these services is not therapeutic, but rather to offer opportunities for people to build social ties within a local community, regardless of their mental health history. During their visits, researchers wrote down field notes whenever possible. These notes were drawn up from memory on a joint document after each visit, so as to complete each other's observations.

Altogether, the research included twenty-nine service users and twenty-seven professionals. Semi-structured interviews and focus groups were audio-recorded and transcribed. Thematic analysis of transcriptions and field notes was carried out using QSR International's NVivo 11 qualitative data analysis software.

Overall, the design and analysis followed groundedtheory procedures in order to approach the social inclusion of people with mental health problems as a social phenomenon, that is, as a process which continually changes in response to evolving conditions. The four interrelated research phases aimed to capture any potentially relevant conditions that contribute to understanding social inclusion, as well as actors' responses to these conditions. In keeping with the methodological framework, this research paid particular attention to participants' experiences and trajectories within and beyond the mental health system; and to the context in which they expressed their perceptions. Furthermore, the broader conditions related to transformations within mental health systems were iteratively incorporated within the explanatory analysis of social inclusion, using our theoretical framework (Luhmann, 2013). For further details regarding grounded theory procedures, the interested reader may refer to Corbin and Strauss (1990). 


\section{Social Inclusion and Social Systems}

General definitions of social inclusion describe processes that aim to create opportunities for people to take part in society (Huxley, 2015). Scholars have approached social inclusion from numerous angles according to research traditions, time, and space (Allman, 2013). Two major dimensions underlie the wide diversity of approaches to social inclusion. On the one hand, material dimensions include access to basic goods, services, and social rights through social systems such as housing, health care, education, and labour. On the other hand, relational dimensions acknowledge experiences of social recognition regarding one's ability to take part in decision-making within communities (Vrooman, Hoff, \& Guiaux, 2015).

The relationships between mental health systems and social inclusion entangle the material and relational dimensions of social inclusion in complex ways. Inclusion within the mental health system does not consistently entail inclusion within other social systems making up society (Wright \& Stickley, 2013). For instance, stigma attached to those seeking mental health care hinders access to employment (Elraz, 2018; Stuart, 2006). Another example involves the medical diagnosis, which sometimes hinders self-definition as a full-fledged member of society through placing mental health service users "in a special role, separat[ing] [them] from the normal and healthy and requir[ing] submission to the authority of specialised personnel" (Illich, 1975, p. 75). The subjective experiences of people with mental health problems could help to disentangle the concept of social inclusion and incorporate its relational dimensions. A more comprehensive approach to the issue could take into account social experiences of inclusion and exclusion, as well as the social production and reproduction of these experiences (Allman, 2013).

Systems Theory (Luhmann, 2013) offers a valuable theoretical framework to tackle the complex relationship between the mental health system and the multiple dimensions of social inclusion. First, it draws a distinction between different types of systems which include social systems and psychic systems. Secondly, it theorizes the process through which these systems interact with one another and relate to their environment.

A system, according to Luhmann (2006), is delineated in terms of the differences with its environment. It is made of elements-such as the father role in a family system or the patient role in a health system, and relations between these elements (Orianne, Collette, Bastin, \& Salmon, 2017, pp. 124-125). Systems are inherently auto-referential since they each create their own elements and relations, which are classified using internally developed references. The mental health system, for instance, is auto-referential as it develops its own references such as the Diagnostic and Statistical Manual of Mental Disorders, in order to classify its patient elements.

A system's environment is composed of other systems. Social systems theory draws a distinction between different types of systems, that is, organic, psychic, and social systems which are equally important. Indeed, a system is always included within another system's environment and neither type of system dominates the other. It follows that psychic systems, or human beings, are not included within social systems. Instead, they are included in social systems' environment and vice versa. Social and psychic systems are based on distinctive modes of operation. A social system is based on communication which raises information and gives meaning through the use of language, schema, and internal references. For instance, the mental health system provides a bio-psychological interpretation of personal problems which may have otherwise been interpreted according to spiritual or cultural references (Abbott, 2014). A psychic system is based on perception or one's ability to pay attention to environmental occurrences. Importantly, communication and perception are interdependent. Indeed, communication cannot go on if not responded to or perceived. It follows that "we then have consciousness in the sense of an orientation towards meaning, on the one hand, and ongoing communication, on the other" (Luhmann, 2013, p. 203).

"Structural coupling" (Luhmann, 2013, pp. 83-101) is the very process which connects psychic systems to social systems. It is based on language and related media enabling systems to know about and to relate to their environment. Through structural coupling, a social system's internal references, elements, and relations are applied to psychic systems, thus reducing or simplifying the latter's strong complexity. For instance, classification systems employed in mental health systems help to establish medical diagnoses which inform treatment and admission procedures. Conversely, reliance on these diagnoses helps people who experience mental health problems to restore order in messy situations, which in turn facilitates relationships with their immediate environment. This simplification process integral to structural coupling is essential for any system's survival. Indeed, a system's assimilation of raw and complex external elements and relations would threaten the boundaries that internal references make it possible to maintain (Dolfsma, Finch, \& McMaster, 2011; Orianne et al., 2017).

Systems Theory helps analyse the relationship between inclusion within the mental health system and social inclusion through two main pathways. First, the mental health system struggles to include complex situations-PCCs-that are resistant to biomedical interpretations, thus threatening the system's boundaries and integrity. These situations typically involve a combination of long-term psychological, social, and economic dimensions. Mental health professionals tend to avoid them, as they are difficult to reduce to medical or psychological explanations (Novella, 2010). Furthermore, people with mental health problems themselves sometimes resist this type of simplification, which holds them responsible for what they may perceive as being dysfunc- 
tions within social systems such as employment or education, rather than dysfunctions within their own psychic systems.

Secondly, inclusion within the mental health system may come into conflict with inclusion within alternative social systems. Different social systems may attribute incompatible meanings to a given situation. For instance, the social systems of mental health care and employment may constrain people with mental health problems to play conflicting roles. In typical cases, this results in hiding mental health problems at the workplace in order to avoid losing a position (Elraz, 2018).

Finally, structural coupling affects both social and psychic systems. Indeed, a difference is produced as soon as the boundaries of two systems meet; in turn, this difference makes a difference (Luhmann, 2013, p. 91). For instance, a psychiatric diagnosis introduces a difference that may affect one's self-perception and relationships with other social and psychic systems.

The empirical material provides further insight into the process of connecting with several social systems based on different modes of operation. It delves into the subjective experiences of inclusion marked by social interactions occurring in different contexts and that contribute to shaping future interactions within the community. Articulating the theoretical framework with this empirical material conveys how two faces of the mental health system contribute to fostering social inclusion, thus introducing empirically-grounded answers to the questions this article raises.

\section{Processes of Social Inclusion: An Empirically-Grounded Approach}

This empirical section is the product of a grounded theory approach to the qualitative material (Corbin \& Strauss, 1990). This approach helps move beyond the material dimensions of social inclusion, such as the availability of the mental health system's services, as it incorporates the relational dimensions of social inclusion. Indeed, the empirical material presented below contributes to conceptualising the relationship between mental health systems and social inclusion, in a way that specifies phenomena "in terms of conditions that give rise to them, how they are expressed through action/interaction, the consequences that result from them, and variations of these qualifiers" (Corbin \& Strauss, 1990, p. 9).

This section introduces PCCs' main features as they relate to organisational challenges within the mental health system. Drawing from similarities between this subgroup and other service users, it then describes the performance of two roles, the patient role and the impatient role, and how they lead to different pathways towards social inclusion. The contextual contributions to these pathways conclude this section, with a focus on the scope of social inclusion.

\subsection{The Struggle of PCCs within Comprehensive Mental Health Systems}

The development of a comprehensive model of mental health care was designed to integrate mental health and social services in a way that could promote patient autonomy, encourage social inclusion, and cease the marginalization of people with mental problems. However, the deinstitutionalisation process has increasingly left out PCCs from the mental health system's services, despite them being particularly affected by social marginalization. The research results highlight certain characteristics of PCCs and mechanisms through which these adverse effects take shape.

First of all, the results highlight the multidimensional nature of PCCs situations as their most important characteristic. They often combine mental health problems with socio-economic vulnerability, substance abuse, or physical disability. These combinations prevent PCCs from meeting the increasingly strict admission criteria established by mental health services, which include hospitals and ambulatory services. Among these criteria, motivation to recover appears as an important obstacle given the complex combination of problems:

The question is, how can they enter the mental health system in the first place? These people have to be highly determined to be admitted. Their problems are multidimensional and severe, but dealing with their mental health is often not their number one priority, this is an important obstacle. (General practitioner)

Furthermore, PCCs describe long-lasting situations due to social isolation and the lack of housing and employment. Those who do receive mental health care face the fragmentation between services and providers, which often leads to revolving doors between hospitals and home environments:

These people have long psychiatric stories....They get discharged from hospital for six months, then they go back, then they get discharged again for a year, and then they go back....They are completely marginalized. (Medical director, psychiatric hospital)

Altogether, our research highlights the increasingly difficult access to mental health services and discontinuity of care for PCCs, a finding which is consistent with a widening body of literature based on many national contexts that promote community models of mental health care (Grard et al., 2015; Novella, 2010). By offering insight into personal experiences of social inclusion, the empirical data considers the relational dimensions of social inclusion and contributes to understanding how transformations within mental health systems shape these experiences. The study sample forms a highly heterogeneous group in terms of medical diagnosis ranging from mood to psychotic disorders; socio-economic sta- 
tus; and degree of involvement with mental health services over their life course. However, all participants have in common that turning to mental health care was subsequent to subjective experiences of exclusion from society. These involve feelings of long-term social isolation, or interpersonal conflicts occurring in various social systems such as family environments, school, or the workplace (Walker et al., 2019). The conceptualisation of these experiences was developed through rigorous analysis of participants' perceptions and interactions in keeping with grounded theory procedures.

\subsection{The Patient Role: Endorsing Medical References, Maintaining Social Systems}

Subjective experiences of social inclusion were expressed through the performance of two roles referred to as the patient role and the impatient role. These roles refer to ideal-types that conceptualise the most salient characteristics of participants' speeches and interactions. In line with a grounded theory approach, the roles are empirically-grounded concepts that enable to set aside individual specificities. This implies that their performance-a set of activities perceived by a set of observers (Goffman, 1959, p. 13)-is not assigned to particular individuals. Instead, specific persons may switch from patient to impatient role performances or the other way around over long periods of time.

Patient roles refer to participants who expressed their personal life narratives based on references typically employed within the mental health system, that is, from a medical perspective which is based on a medical diagnosis. Patient roles were most often performed by people having experienced long-term and uninterrupted trajectories within the mental health system, and who genuinely integrated the way this system interpreted episodes of mental distress. For instance, Emily refers to her physician's diagnosis to make sense of problems she encountered at work that led her to enter the mental health system:

In the beginning, I was aware that I didn't know where I was going professionally, I was lost, and I didn't know what was wrong with me. When the doctor told me I was having a burnout, at least I could put a name on what it was....Then, it's a matter of accepting the situation, even just the word "hospital." I learned so much during those years in the psychiatric hospital.

For patient role performers, subjective feelings of social inclusion arise when significant others (Goffman, 1967) also acknowledge the medical diagnosis associated with their mental distress and adapt their attitudes accordingly. Emily's experience illustrates how references to a medical diagnosis can importantly mediate social relationships and eventually lead to feelings of social support and inclusion:
Many people saw me as a strong woman, so they were surprised to find out I was having a burnout....But my body reached its limits, and I had to wait for people to accept that before receiving their support.

The mental health system appears as an important mediator for patient role performers to experience subjective feelings of social support and inclusion. However, further evidence suggests that this form of social inclusion remains limited to the mental health system and empathetic significant others. Indeed, patient role performers importantly struggle to expand subjective feelings of social inclusion beyond the mental health system's boundaries, such as the workplace in Emily's case:

After two years [in the hospital], I wanted to try out some volunteer work, to challenge myself at work again....Since then, I've been involved in projects here at [association], I arrived as a mental health service user....At times, I'm very happy with volunteering two days a week but sometimes it's a problem because it's just volunteer work, not a real job! I mean it feels like a real job to me, but legally speaking, it's not a real job. I think they could pay me for what I do.

The findings highlight patient role performers' struggle to find a regular job that recognizes their status as a mental health service user, for instance, as a service user representative. Moreover, they reveal experiences of discrimination within other social systems on being primarily identified as people with mental health problems (Walker et al., 2019). Altogether, this data suggests that subjective feelings of social inclusion beyond the mental health system's boundaries and significant others remains a challenge for patient role performers.

\subsection{The Impatient Role: Challenging System Boundaries}

Performance of impatient roles describe alternative processes of social inclusion. Indeed, although impatient role performers also seek recognition and acceptance within multiple social systems, they do not want the mental health system to interfere. The following extract illustrates the performance of an impatient role, that seeks to achieve social inclusion on the basis of professional qualifications, as opposed to a medical diagnosis:

That's what we need, a place in the community, where we're accepted the way we are....It could also be a job! [Psychiatry] treated me like I was crazy, but I wasn't crazy at all!...My civil status states that I'm disabled, a psychotic patient, but I don't want it to be that way. It's about time I live my life and society accepts me as the civil engineer that I am!

This extract illustrates how impatient role performers are opposed and irritated with the medicalisation of their life narratives. In fact, they often question medical ref- 
erences and express strong reactions regarding their use within the mental health system:

They use a stupid system of classification. If you roughly look or behave like someone else, you're considered the same person. So to me, it doesn't make any sense. Every human being is different, has a different story and holds different beliefs. They label us so they can classify us and turn us into the victims of pharmaceutical companies. We're just pawns, they use us to create and maintain their own system!

Impatient role performers were mostly PCCs encountered in alternative services during the ethnographic fieldwork. A detailed description of these services and of their history furthers our understanding of how and why they attract this population.

Alternative services are not formally included within the mental health system, and were often created on the initiative of service users themselves and their relatives. They can be described as inclusive spaces which voluntarily de-emphasize both social and diagnostic categories in everyday interactions. These spaces are open to the public and covertly intend to integrate former or current mental health service users within mainstream society. They are mostly centrally situated and accessible to anyone passing by, regardless of their mental health history. Alternative services do not provide psychiatric or medical treatment, but allow for opportunities to develop ties within a local community through the organization of collective activities.

The coordinator of an alternative service explains its history and underlying philosophy. This association developed in the 1960s, during the anti-psychiatric movement. It aimed to find a middle ground between discharging patients without providing any alternative, and reproducing a different type of "total institution" (Goffman, 1961) providing all-inclusive care. Gradually, this association drifted away from the anti-psychiatric movement: "We used to identify as social activists, but now, we want to contribute to social change by being part of society" (interview with the coordinator of an alternative service). In fact, the association developed ties with institutions that are included in the mental health system and that sometimes introduce PCCs to this alternative service. This particularly occurs when PPCs have experienced a long and conflicting journey within the mental health system and are willing to leave this system. The coordinator describes the association as de-emphasizing psychiatric categories and more generally, as blurring the boundaries between patients, professionals and regular citizens. All visitors are considered members as soon as they step through the door, which creates opportunities to foster heterogeneous communities:

This form of socialization within heterogeneous communities has important implications for the social inclusion of people with mental health problems. Altern- ative services help them develop alternative aspects of their identity through the co-construction of collective events based on personal or cultural interests. People with a long journey within the mental health system are so used to talking about their mental health problems, and to identifying as a 'patient.' We ask them to tell us another story! What do you want to do?

This approach seems to play an important role in limiting self-identification as a patient and fostering feelings of belonging to a community that goes beyond the mental health system. A participant reflects on the difference between a day hospital and the alternative service he has been going to this past year:

I ask myself more and more, is it really that different? It also keeps you busy, gets you up in the morning, forces you to get dressed, meet other people, get together and chat....Except there's no medical jargon around it all, that's one thing out of the way. If I had to tell the difference, I'd say we engage in activities but they're not medicalised. We're not seen as patients!...I'm seen like a man.

Alternative services support a personal transformation process in which 'patients' are given opportunities to explore alternative roles. This process simultaneously contributes to removing the stigma of mental health. Indeed, alternative services de-emphasize social and diagnostic categories, and visitors cannot tell apart mental health service users from other visitors belonging to disparate social worlds. They are all primarily focused on the collective events which turn into intermediary objects. Stigma is also reduced through exposing the general public to non-conventional behaviours. A field note extract illustrates these parallel processes:

The front door of [alternative service] situated in central Brussels is wide open. Though a small number of visitors are sitting and chatting on the sunny terrace as in other neighbouring cafés, most visitors are inside listening to an amateur piano player. Upon hearing the piano from the pavement, a young couple enters the space and walks towards the bar where they order two beers. The bartender, who the researcher identified as a mental health service user on previous occasions, serves the order while simultaneously displaying notable behaviours: repeatedly walks back and forth from wall to bar, mumbles to herself, slowly pours the beer bottles into glasses whilst spilling some onto the bar surface. The bartender informs the couple the beers are at an open price and the man hands over some coins. Surprised, the woman asks her partner why that is: "Dunno, I think this place is the association I heard about, something to do with mental health or the homeless." The couple moves onto watching the piano player and quietly comments on how talented she is. 
Altogether, alternative spaces offer occasions for mental health service users to explore different roles through exposure to social interactions within the community. The co-construction of events within communities made of different social worlds mediates this process of inclusion within mainstream society. This contrasts with patient role performances, which limit social inclusion to a reduced network of mental health professionals, peers, and empathetic others that communicate using individual medical references. Finally, alternative services support a process of social inclusion that helps its members adapt to open and continuously transforming social systems where people come and go. A coordinator explains this requires minimal intrusion into people's lives. Members are encouraged to select from whichever references they need to make sense of the world and of where they stand:

Not being intrusive means openness to any resources available within the community. Indeed, these people were often captive within the mental health system and want to get out of it, so we don't want to replace this system with another one...by imposing certain references. The alternative is to open doors and resources that exist within the community. We offer our members support as they explore these different resources, but we don't want to tell them what to do with them and where to go, nor do we want them to depend on us.

\section{Discussion}

This article addresses the challenge of achieving the social inclusion of people with mental health problems in the context of mental health care deinstitutionalisation. It employs a broad definition of social inclusion which acknowledges several dimensions, including access to services available within a community but also experiences of social involvement as full-fledged members of society.

The reorganization of mental health systems across Europe aims to promote personal autonomy and to support social inclusion for all. However, this process has substantially hindered access to services available within the mental health system over the past few decades, particularly for PCCs. Furthermore, those who do access existing services do not consistently experience feelings of social involvement within their community and of belonging to society. In order to address these complexities, this article raises two main questions. First, how do contemporary mental health systems contribute to the process of social inclusion for people with mental health problems? Secondly, how can PCCs navigate towards social inclusion, in the context of their increasing exclusion from the mental health system's services?

To address these questions, this article employed a theoretical framework that could support an in-depth discussion about social systems (such as the mental health system), and a conception of social inclusion which incorporates its relational dimension. Luhmann's (2013) Systems Theory was highly useful in this respect. According to Systems Theory, social systems develop and survive by maintaining a difference with their environment, which is made of other systems and human beings, who are referred to as psychic systems. Each system maintains its difference through communication, an essential mode of operation to establish relationships with its environment. A mode of operation implies the selection and simplification of events occurring within the system's environment, a process referred to as specification, which relies on internally created categories. Admission procedures are examples of categories created and employed by the mental health system to interpret situations occurring in its environment. Specification is integral to a system's survival. Indeed, should several social systems assign the same meaning to a given event, differences would dissolve and systems would be threatened.

Systems Theory helps understand the increasing exclusion of PCCs from the mental health system, as further evidenced by the empirical material. Indeed, while they combine biological, social, and psychological dimensions, PCCs easily resist the mental health system's specification which is rooted in biomedical interpretations. Their exclusion from this system helps maintain its integrity. Furthermore, if a system can only assign a specific meaning to a given situation, it poses a challenge for this situation to be included in multiple systems simultaneously (e.g., in employment and mental health care).

A grounded theory approach to the subjective experiences of people with mental health problems shed light on the process of establishing relationships between themselves, conceived as psychic systems, and their social environment. This analysis highlighted the performance of two roles: the patient role and the impatient role. Patient role performers learn to interpret their personal situations by relying on medical discourse. They identify with the medical diagnosis entitling them to mental health care. Although this role may offer opportunities to resolve social ties with empathetic others, it is simultaneously discriminated against by certain individuals and within certain social systems such as employment. Conversely, patient role performers sometimes self-exclude from these social systems by doubting this role is compatible with those required by other social systems. By and large, although inclusion within the mental health system can foster a subjective sense of social inclusion, it is often limited to the mental health system's boundaries.

In contrast, impatient role performers do not believe that medical references can account for their personal situations, nor do they want the mental health system to mediate their social inclusion. They seek opportunities to explore alternative references to make sense of the world and of their situation, and appreciate the support from alternative services during this process. Importantly, impatient roles support processes of inclu- 
sion that are not limited to the mental health system's boundaries, but that expand towards society at large.

The mental health system's mode of operation, or specification, contributes to answering the first question raised in this article. Indeed, it helps understand the limits of the mental health system's contribution to social inclusion. By fully endorsing this form of specification, people with mental health problems have very limited possibilities to develop social ties beyond the mental health system, that is, outside of mental health professionals, empathetic significant others or peers. Opportunities to explore or re-establish connections with other social systems, this article argues, is integral to processes of inclusion within broader communities.

The empirical material highlights alternative services' central role in facilitating this process of inclusion within society at large, through supporting connections between several psychic and social systems. The contextual and interactional elements described in the empirical section help understand how these services facilitate this process.

The coordinators conveyed alternative services' ability to self-define by marking a difference with their environment, which includes the mental health system. The essential difference lies in dissolving the status of patient which gives rise to an inclusive status of member, a reverse operation mode to specification which could be referred to as despecification. This difference is reproduced in alternative services' material and non-material characteristics-accessibility, openness, and diversity of social and cultural activities. It is further supported by members themselves. Indeed, they express deep feelings of being perceived as human beings taking part in society, where sitting down to have a chat appears in the foreground. Finally, despecification was observed in action which made it possible to assess its impact on social inclusion. Indeed, considering PCCs as fully-fledged members and taking part in social and cultural activities yields social ties between PCCs and other citizens. In this context, PCCs are given the opportunity to receive social acknowledgement for alternative attributes such as a particular talent, despite their mental health problem.

Based on Systems Theory, alternative services can be described as forming a social system of their own, since they communicate their difference which its members and other citizens perceive. As any social system, this system creates a difference which survives and persists through material and non-material communication. Unlike other social systems, inclusion within it does not rely on specification, but rather, on despecification. This system can be said to be on the flip side of the mental health system.

The answer to our second question regarding the pathways towards social inclusion of PCCs lies precisely in despecification. By bringing people together regardless of their social status around a social or a cultural activity, alternative services form a system that offers them the possibility to connect with society at large.
Altogether, this analysis disentangles the social inclusion of people with mental health problems. It replaces a dichotomous approach according to which a mental health service user is either included or excluded from society. Drawing from the empirically-grounded concepts of patient and impatient roles, the connections between psychic and social systems are unsettled and dynamic; they depend on the relationship between one's perception of a complex personal situation, and the meaning which a social system assigns to the same situation. Examining these relationships shed light on how they are created, restrained, or stimulated through specification in mental health systems, and despecification in alternative services.

Based on this analysis, the complementarities between the mental health system and alternative services seem central to achieving social inclusion beyond the mental health system and towards society at large. Indeed, the alternation between specification, within the mental health system, and despecification within alternative services, enables to regularly suspend roles and social positions, thus making room to explore new roles in several social systems.

\section{Conclusion}

The argument developed throughout this article suggests policy recommendations. Incorporating the relational dimensions of social inclusion highlights the complementarity between the mental health system and a system made up of alternative services. Both systems supplement each other at different times in order to mediate between people with mental health problems and social environments. This mirrors the trajectories of those who alternate between patient and impatient role performances at different points in time. The complementarities between both systems are deeply intertwined with their contrasting modes of operation-specification and despecification, which they must each maintain in order to survive. On this basis, a suggestion for effective public action in the field of mental health would be to strike a balance between both systems in terms of political and financial support, while maintaining their modes of operation. Furthermore, intensifying the relationships between these social systems would enable service users to benefit from their complementarities.

A priori, this article's analysis is limited to the Brussels Region where the research drawn on was carried out. However, the deinstitutionalisation process and the challenges for PCCs are observed in many OECD countries. Thus, future research could initiate an international comparative analysis focused on the articulation between social systems and the trajectories of PCCs.

\section{Acknowledgments}

The authors thank the stakeholders of the Belgian mental health system and the people encountered within al- 
ternative spaces for their trust. They thank the research sponsors, the Common Community Commission of the Brussels Region and the Social and Health Observatory. They thank their colleagues, particularly Pablo Nicaise, for their support during the research process. Finally, they thank Patricia Walker for proofreading this article.

\section{Conflict of Interests}

The authors declare no conflict of interests.

\section{References}

Abbott, A. (2014). The system of professions: An essay on the division of expert labor. Chicago, IL: University of Chicago Press.

Allman, D. (2013). The sociology of social inclusion. SAGE Open, 3(1), 1-16.

Bergeron, H., \& Castel, P. (2016). Les habits neufs du néoinstitutionnalisme? La redécouverte de l'ordre mésologique et de l'agency [Recent developments in organizational institutionalism]. L'Année Sociologique, 66(1), 31-72.

Corbin, J. M., \& Strauss, A. (1990). Grounded theory research: Procedures, canons, and evaluative criteria. Qualitative sociology, 13(1), 3-21.

de Munck, J., Genard, J.-L., Olgierd, K., Vrancken, D., \& Delgoffe, D. (2003). Santé mentale et citoyenneté: Les mutations d'un champ de l'action publique [Mental health and citizenship: Changes in a field of public action]. Ghent: Academia Press.

Dolfsma, W., Finch, J., \& McMaster, R. (2011). Identifying institutional vulnerability: The importance of language, and system boundaries. Journal of Economic Issues, 45(4), 805-818.

Elraz, H. (2018). Identity, mental health and work: How employees with mental health conditions recount stigma and the pejorative discourse of mental illness. Human Relations, 71(5), 722-741.

Federal Service for Public Health. (2010). Guide vers de meilleurs soins en santé mentale par la réalisation de circuits et de réseaux de soins [Guide towards better mental health care through the creation of care pathways and networks]. Brussels: Federal Service for Public Health. Retrieved from http://www. psy107.be/files/Bruxelles.pdf

Feys, J.-L. (2017). Les fondements constructivistes de l'antipsychiatrie. L'information Psychiatrique, 93(6), 457-463.

Gerkens, S., Farfan-Portet, M.-I., Desomer, A., Stordeur, S., de Waroux, M., van de Voorde, C., . . . Leonard, C. The Belgian health system in 2010 (KCE Reports 138C. D/2010/10.273/61). Brussels: Belgian Health Care Knowledge Centre.

Goffman, E. (1959). The presentation of self in everyday life. Garden City, NY: Doubleday \& Company.

Goffman, E. (1961). Asylums: Essays on the social situation of mental patients and other inmates. Garden
City, NY: Doubleday \& Company.

Goffman, E. (1967). Interaction ritual: Essays in face to face behavior. Garden City, NY: Doubleday \& Company.

Grard, A., Nicaise, P., \& Lorant, V. (2015). Evaluation de la réforme "Vers de meilleurs soins en santé mentale" Résultats 2014 [Evaluation research on the mental health care reform for adults in Belgium-Results for 2014]. Acta Psychiatrica Belgica, 115(1), 40-49.

Hermans, M. H. M., de Witte, N., \& Dom, G. (2012). The state of psychiatry in Belgium. International Review of Psychiatry, 24(4), 286-294.

Huxley, P. (2015). Introduction to "Indicators and Measurement of Social Inclusion." Social Inclusion, 3(4), 50-51.

Illich, I. (1975). Limits to medicine. London: Boyars Publishers.

Luhmann, N. (2006). System as difference. Organization, 13(1), 37-57.

Luhmann, N. (2013). Introduction to systems theory. Cambridge: Polity Press.

Novella, E. J. (2010). Mental health care and the politics of inclusion: A social systems account of psychiatric deinstitutionalization. Theoretical Medicine and Bioethics, 31(6), 411-427.

Orianne, J.-F., Collette, F., Bastin, C., \& Salmon, É. (2017). La maladie d'Alzheimer sous l'angle de la théorie générale des systèmes [Alzheimer's disease from the perspective of systems theory]. Revue de Neuropsychologie, 9(2), 109-117.

Stuart, H. (2006). Mental illness and employment discrimination. Current Opinion in Psychiatry, 19(5), 522-526.

Sturdy, S., Freeman, R., \& Smith-Merry, J. (2013). Making knowledge for international policy: WHO Europe and mental health policy, 1970-2008. Social History of Medicine, 26(3), 532-554.

Thunus, S. (2015). The system for addressing personal problems-From medicalisation to socialisation: Shifts in Belgian psychiatric and mental health institutions (Unpublished Doctoral dissertation). University of Liège, Liège, Belgium. Retrieved from https:// orbi.uliege.be/bitstream/2268/180914/1/thesis \%20SThunus.pdf

Thunus, S., \& Schoenaers, F. (2017). How does policy learning occur? The case of Belgian mental health care reforms. Policy and Society, 36(2), 270-287.

Verhaegen, L. (1987). The evolution of Belgian psychiatry. International Journal of Mental Health, 16(1/2), 42-57.

Vrooman, J. C., Hoff, S. J. M., \& Guiaux, M. (2015). Descendants of hardship: Prevalence, drivers and scarring effects of social exclusion in childhood. Social Inclusion, 3(4), 76-97.

Walker, C., Nicaise, P., \& Thunus, S. (2019). Parcours. Bruxelles: Evaluation qualitative du système de la santé mentale et des parcours des usagers dans le cadre de la réforme Psy 107 en Région de Bruxelles- 
Capitale [Life and care trajectories within the mental health system in Brussels, Belgium: A qualitative evaluation research in the context of a national mental health care reform] Brussels: Health and Society Institute, UCLouvain.

World Health Organization. (2018). Mental health, human rights and standards of care: Assessment of the quality of institutional care for adults with psychosocial and intellectual disabilities in the WHO European region. Copenhagen: World Health Organization. Wright, N., \& Stickley, T. (2013). Concepts of social inclusion, exclusion and mental health: A review of the international literature. Journal of Psychiatric and Mental Health Nursing, 20(1), 71-81.

\section{About the Authors}

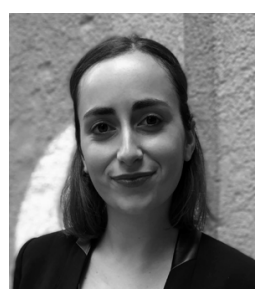

Carole Heather Walker is a PhD Student at the Health and Society Institute and Teaching Assistant at the Public Health Faculty of UCLouvain, Belgium. Having earned an MA in Psychology, she began her research activities in 2017 using qualitative methods and doing ethnographic fieldwork. Her initial research focused on social inclusion in the context of mental health care organisation. Her doctoral dissertation will examine the reciprocal influences between migration flows and health systems in urban contexts, particularly access to health care for socio-cultural minority groups.

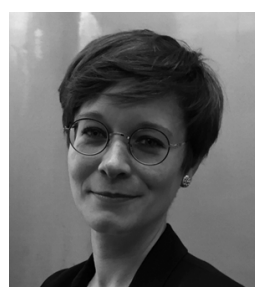

Sophie Thunus is an Assistant Professor of Health Services Management at the Public Health Faculty of UCLouvain, Belgium. She holds a PhD in Social and Political Science (ULiège, Belgium, 2015) and is experienced in qualitative research on health and mental health policy design and implementation. Her publications raise questions relating to change in the nature and distribution of roles, power and knowledge among the health care system's stakeholders. Her main research interests focus on the role of "meetings" in mediating changes in social identities and systems. 